\title{
Molecular Coordination, Structure, and Stability of Metal-Polyphosphate Complexes Resolved by Molecular Modeling and X-ray Scattering: Structural Insights on the Biological Fate of Polyphosphate
}

\author{
Yeonsoo Park ${ }^{1,2}$, Christos D. Malliakas ${ }^{3}$, Qing Zhou ${ }^{4,5}$, April Z. Gu4 Ludmilla Aristilde $^{1,2, *}$ \\ ${ }^{1}$ Department of Biological and Environmental Engineering, College of Agriculture and Life \\ Sciences, Cornell University, Ithaca, NY 14853 USA \\ ${ }^{2}$ Department of Civil and Environmental Engineering, McCormick School of Engineering and \\ Applied Science, Northwestern University, Evanston, IL 60208 USA \\ ${ }^{3}$ Department of Chemistry, Northwestern University, Evanston, IL 60208 USA \\ ${ }^{4}$ Department of Civil and Environmental Engineering, College of Engineering, Cornell University, \\ Ithaca, NY 14853 USA \\ ${ }^{5}$ School of the Environment, Nanjing University, Nanjing, Jiangsu Province 210023, China
}

(*correspondence: ludmilla.aristilde@northwestern.edu)

\section{SUPPORTING INFORMATION}

Table S1

Table S2

Figure S1

Figure S2

Figure S3

Details of PDF calculation 
Table S1. Comparison of essential bond lengths $(\AA)$ and angles $\left({ }^{\circ}\right)$ within the Ca-pyrophosphate structure from the simulation (average \pm standard deviation) and the reference (Gras et al. 2016). Atom numbered according to the reference.

\begin{tabular}{lccc}
\hline Bond length & Simulation & Reference & \% difference \\
\hline O1 - P9 & $1.593 \pm 0.040$ & 1.492 & 6.8 \\
O8 - P10 & $1.580 \pm 0.036$ & 1.581 & 0.0 \\
O8 - P9 & $1.612 \pm 0.045$ & 1.688 & 4.5 \\
O7 - P10 & $1.517 \pm 0.037$ & 1.525 & 0.5 \\
O3 - P9 & $1.480 \pm 0.028$ & 1.510 & 2.0 \\
O4 - P9 & $1.635 \pm 0.041$ & 1.551 & 5.4 \\
O5 - P10 & $1.581 \pm 0.039$ & 1.533 & 3.1 \\
O6 - P10 & $1.477 \pm 0.029$ & 1.501 & 1.6 \\
Ca11 - O1 & $2.585 \pm 0.074$ & 2.372 & 9.0 \\
O5 - Ca2 & $2.772 \pm 0.086$ & 2.343 & 18.3 \\
\hline
\end{tabular}

\begin{tabular}{lccc}
\hline Bond angle & Simulation & Reference & \% difference \\
\hline O1-P9-O3 & $101.8 \pm 4.0$ & 113.4 & 10.2 \\
O1-P9-O4 & $111.2 \pm 7.6$ & 112.7 & 1.4 \\
O1-P9-O8 & $115.9 \pm 3.4$ & 107.0 & 8.3 \\
O4-P9-O8 & $113.3 \pm 9.0$ & 116.4 & 2.7 \\
O3-P9-O4 & $100.2 \pm 4.6$ & 104.2 & 3.8 \\
O3-P9-O8 & $112.1 \pm 5.4$ & 101.5 & 10.5 \\
P9-O8-P10 & $97.2 \pm 3.6$ & 128.3 & 24.2 \\
O5-P10-O7 & $94.6 \pm 5.6$ & 113.3 & 16.5 \\
O5-P10-O6 & $109.0 \pm 4.9$ & 106.2 & 2.7 \\
O5-P10-O8 & $107.3 \pm 7.1$ & 106.0 & 1.2 \\
O6-P10-O7 & $50.4 \pm 8.1$ & 114.7 & 56.1 \\
O7-P10-O8 & $100.1 \pm 12.0$ & 106.4 & 5.9 \\
O6-P10-O8 & $116.8 \pm 24.0$ & 109.9 & 6.2 \\
\hline
\end{tabular}


Table S2. Coordination distance (average \pm standard deviation, $\AA$ ) and number (CN) of the metal (M)-polyphosphate (polyP) complexes. The reported values have been recalculated based on the values reported in Table 1. Instead of calculating the distance and $\mathrm{CN}$ up to the global minimum as in Table 1, the values here are recalculated up to the radial distance at which the $\mathrm{CN}$ value was closest to the rounded up whole number of the $\mathrm{CN}$ values reported in Table 1 .

\begin{tabular}{|c|c|c|c|c|c|c|}
\hline \multirow{2}{*}{ M } & \multicolumn{2}{|c|}{ M-ligand O } & \multicolumn{2}{|c|}{ M-bridging $\mathrm{O}$} & \multicolumn{2}{|c|}{$\mathrm{M}$-water $\mathrm{O}$} \\
\hline & Distance & $\mathrm{CN}$ & Distance & $\mathrm{CN}$ & Distance & $\mathrm{CN}$ \\
\hline \multicolumn{7}{|c|}{ M-polyP-10 complexes } \\
\hline $\mathrm{Na}^{+}$ & $2.58 \pm 0.56$ & 2.07 & $2.73 \pm 0.55$ & 1.01 & $2.73 \pm 0.51$ & 3.07 \\
\hline $\mathrm{K}^{+}$ & $2.70 \pm 0.46$ & 1.99 & $2.88 \pm 0.49$ & 1.01 & $2.76 \pm 0.35$ & 2.06 \\
\hline $\mathrm{Ca}^{2+}$ & $2.43 \pm 0.37$ & 1.96 & $3.09 \pm 0.75$ & 2.03 & $2.40 \pm 0.25$ & 0.97 \\
\hline $\mathrm{Mg}^{2+}$ & $1.98 \pm 0.32$ & 2.01 & $2.64 \pm 0.70$ & 2.04 & $2.52 \pm 0.42$ & 0.96 \\
\hline \multicolumn{7}{|c|}{ M-polyP-30 complexes } \\
\hline $\mathrm{Na}^{+}$ & $2.79 \pm 0.68$ & 3.00 & $2.85 \pm 0.65$ & 1.03 & $2.46 \pm 0.35$ & 1.97 \\
\hline $\mathrm{K}^{+}$ & $2.76 \pm 0.49$ & 1.99 & $3.24 \pm 0.60$ & 0.96 & $2.67 \pm 0.34$ & 1.94 \\
\hline $\mathrm{Ca}^{2+}$ & $2.52 \pm 0.46$ & 1.98 & $2.97 \pm 0.51$ & 1.00 & $2.25 \pm 0.20$ & 0.97 \\
\hline $\mathrm{Mg}^{2+}$ & $2.43 \pm 0.58$ & 2.98 & $2.46 \pm 0.53$ & 1.02 & $2.46 \pm 0.46$ & 1.98 \\
\hline
\end{tabular}
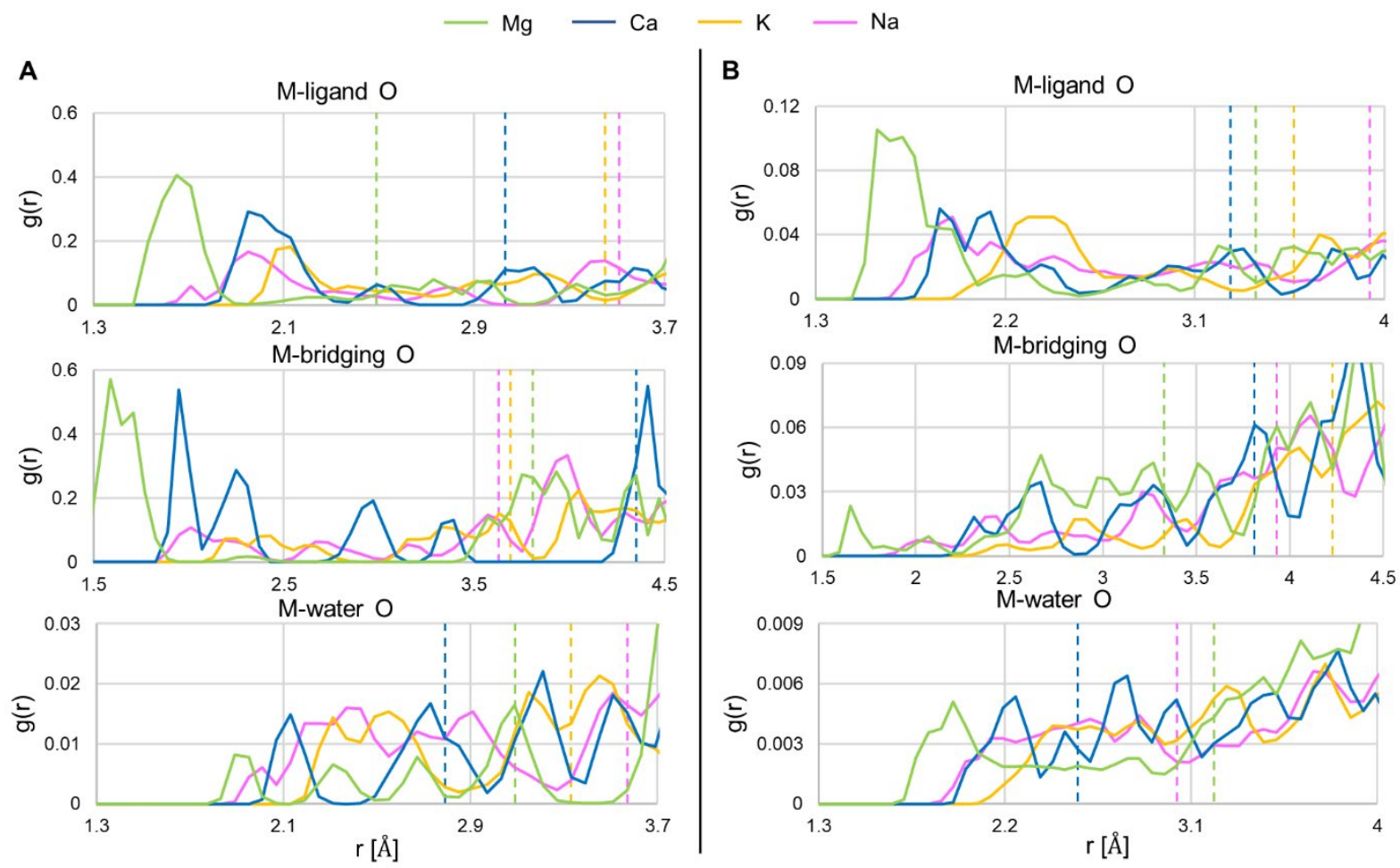
Figure S1. Radial distribution functions (RDF, $g(r)$ ) of different $O$ groups within the metal (M)polyphosphate (polyP) complexes: (A) polyP-10 and (B) polyP-30. The dotted lines mark the radial distance at which the calculated coordination number (CN) was closest to the rounded up whole number. Color code: $\mathrm{Na}$ (pink), K (yellow), Ca (blue), and Mg (green).

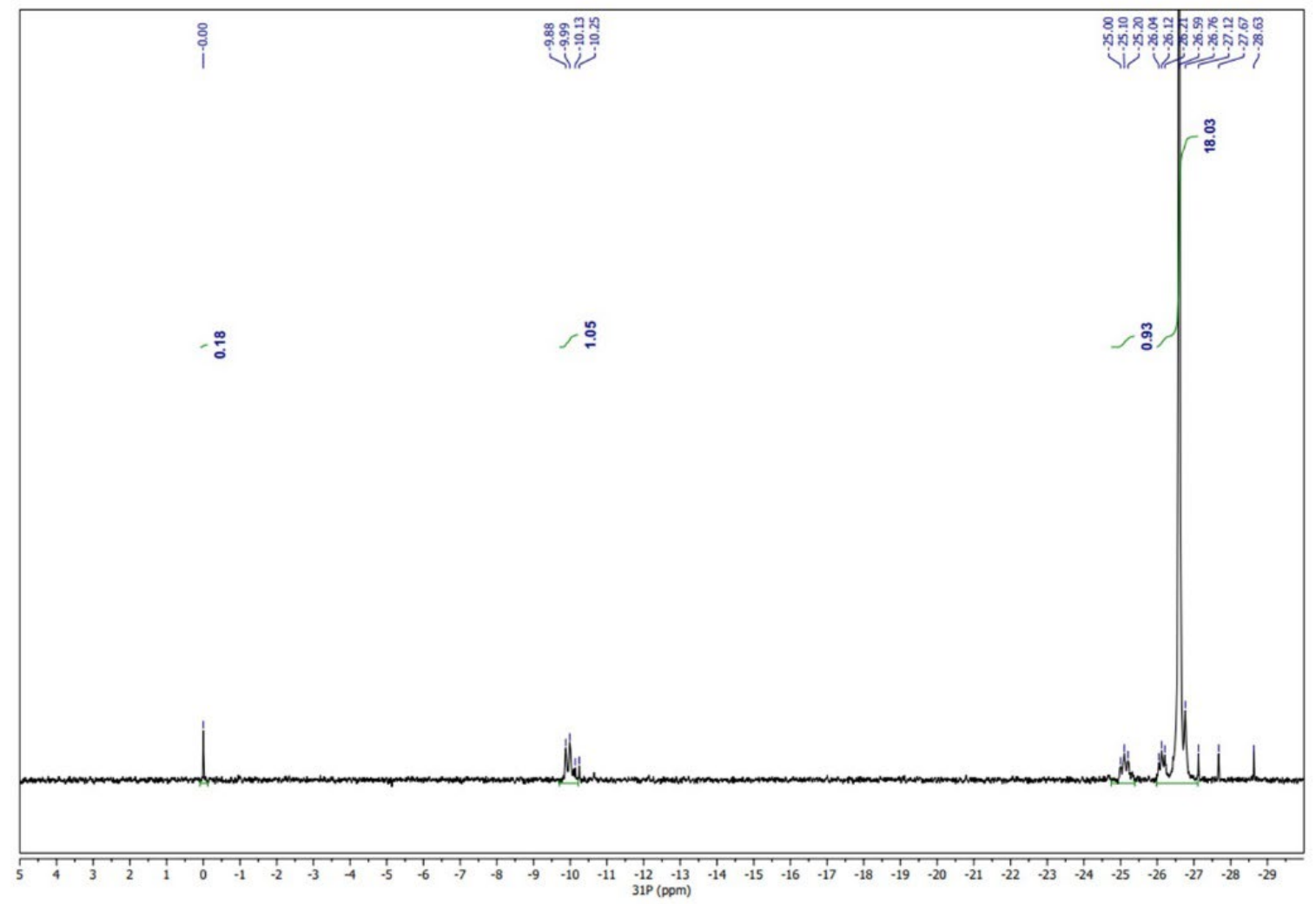

Figure S2. ${ }^{31} \mathrm{P}-\mathrm{NMR}$ results of the synthesized polyP-30. $100 \mathrm{mg}$ of sodium polyP-30 was dissolved in $0.9 \mathrm{~mL} 1 \mathrm{M} \mathrm{NaOH}$ and $0.1 \mathrm{~mL} \mathrm{D}_{2} \mathrm{O}$. The sample was analyzed by a Bruker $400 \mathrm{MHz}$ NMR spectrometer using $1.6 \mathrm{~s}$ acquisition time, $2.4 \mathrm{~s}$ repetition rate, $65^{\circ}$ acquisition angle and 110 scans. 


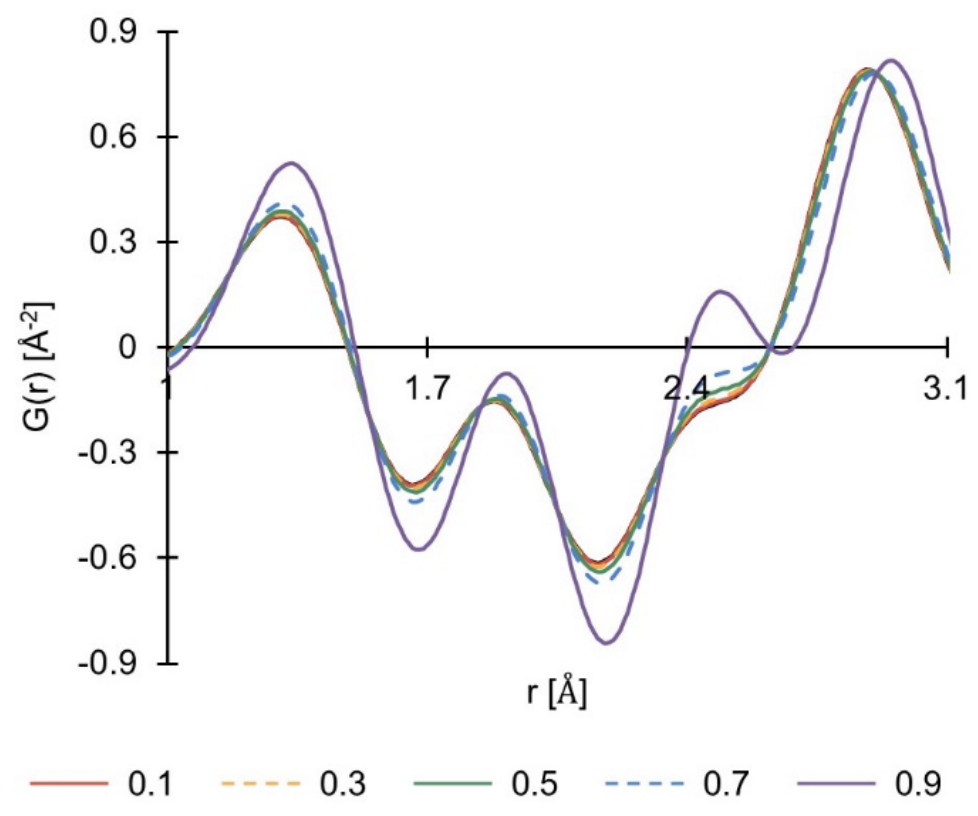

Figure S3. Pair distribution function (PDF, G(r)) obtained from X-ray scattering, both without (black) and with various reference solution correction factors $(0.1,0.3,0.5,0.7$, and 0.9$)$. 


\section{Details of the pair distribution function (PDF) calculation}

The experiments were straightforward X-ray powder diffraction measurements. From the coherent part, $I^{\operatorname{coh}}(Q)$, of the measured total diffracted intensity of the material we find the total scattering structure function, $S(Q)$

$$
S(Q)=\frac{I^{\operatorname{coh}}(Q)-\sum c_{i}\left|f_{i}(Q)\right|^{2}}{\left|\sum c_{i} f_{i}(Q)\right|^{2}}+1
$$

where the coherent intensity is corrected for background and other experimental effects and normalized by the flux and number of atoms in the sample. Here, $c_{i}$ and $f_{i}$ are the atomic concentration and X-ray atomic form factor, respectively, for the atomic species of type $i$. Momentum transfer, $Q$, is given by

$$
Q=4 \pi \sin \vartheta / \lambda
$$

By Fourier transforming the expression $Q[S(Q)-1]$ we have

$$
G(r)=(2 / \pi) \int_{Q=0}^{Q_{\max }} Q[S(Q)-1] \sin (Q \cdot r) d Q
$$

where $G(r)$ is the atomic pair distribution function which is also defined as

$$
G(r)=4 \pi \cdot r\left[\rho(r)-\rho_{0}\right]
$$

where $\rho_{0}$ is the average atomic number density, $\rho(r)$ is the atomic pair-density and $r$ is a radial distance. The function $G(r)$ gives information about the number of atoms in a spherical shell of unit thickness at a distance $r$ from a reference atom. Finally, the experimental $G(r)$ can be compared and refined against a theoretical $G(r)$ from a structural model given by 


$$
G(r)+4 \pi \cdot r \cdot \rho_{0}=\frac{1}{r} \sum_{v} \sum_{\mu} \frac{f(0)_{v} f(0) \mu}{\langle f(0)\rangle^{2}} \delta\left(r-r_{v \mu}\right)
$$

In theory, $Q_{\max }$ from equation (3) should by infinite but experimentally is always a finite number. In order to have good resolution, values of $Q>20 \AA^{-1}$ are desirable therefore short wavelengths are necessary according to equation (2).

Unlike a conventional X-ray or neutron powder diffraction experiment, the PDF analysis treats both the Bragg and diffuse scattering on an equal basis. Because the data analysis does not presume any periodicity, the technique is very useful for examining samples if distortions found in a specific single crystal are representative of the total bulk of the sample. 